\title{
Propuesta de sistema de control de gestión para optimizar los procesos del departamento de compras y ventas
}

\author{
Proposal for a management control system to optimize the processes of the \\ purchasing and sales department
}

\author{
Alfredo Castellano \\ alfredocastellanocarreyo@gmail.com \\ ORCID: 0000-0001-7994-0668
}

Universidad de Carabobo, Valencia - Venezuela
Deibis Madroñero

deibismadroñero@gmail.com ORCID: 0000-0002-4649-104X

Universidad de Carabobo, Valencia - Venezuela

Artículo recibido febrero 2020 / Arbitrado en marzo 2020 / Aceptado junio 2020 / Publicado en julio 2020

\begin{abstract}
RESUMEN
La presente investigación tiene por objetivo proponer un sistema de control de gestión para optimizar los procesos operativos del departamento de compras y ventas de la empresa. Metodológicamente la investigación fue de tipo descriptiva, modalidad proyecto factible, diseño de campo. El estudio fue complementado con fuentes bibliográficas-documentales y la observación directa. La recolección de datos fue a través de un cuestionario de preguntas dicotómicas aplicado a una muestra intencional constituidas por los empleados del departamento de compras y ventas. Los datos permitieron concluir que existe deficiencia en los procesos operativos asociados al departamento, la ausencia de normas, políticas y procedimientos que permitan controlar los procesos en cuanto al requerimiento de materia prima, la incapacidad de la empresa de concretar ventas de productos terminados; situaciones que permitió configurar un sistema de control para el adecuado manejo en los procesos operativos asociados a las compras y ventas de la empresa.
\end{abstract}

Palabras clave: Compras; gestión; procesos; sistema; ventas

\begin{abstract}
The objective of this research is to propose a management control system to optimize the operational processes of the company's purchasing and sales department. Methodologically the research was descriptive, feasible project modality, field design. The study was complemented with bibliographic-documentary sources and direct observation. The data was collected through a dichotomous questionnaire applied to an intentional sample made up of employees from the purchasing and sales department. The data allowed concluding that there is a deficiency in the operational processes associated with the department, the absence of norms, policies and procedures that allow controlling the processes regarding the requirement of raw materials, the inability of the company to make sales of finished products; situations that allowed configuring a control system for proper management in the operational processes associated with the company's purchases and sales.
\end{abstract}

Keywords: Purchases; management; processes; system; sales 


\section{INTRODUCCIÓN}

A lo largo de la historia las organizaciones empresariales han sido una parte importante en la economía de un país, porque además de obtener un valor agregado para remunerar a los factores que participan, tiene como finalidad la producción, a través de la compra y venta de materiales e insumos para diseñar, construir, realizar o fabricar ropa, calzados, entre otros, para satisfacer las necesidades de la sociedad, por consiguiente debe existir un buen funcionamiento en los procesos; pues estos generan la renta de una organización y su actividad principal, razón por la cual se debe tomar en cuenta cada mecanismo y gestión que permita su óptimo funcionamiento.

Las organizaciones empresariales cuentan con herramientas de control que les permite evaluar constantemente su posición económica, además de permitir una toma de decisiones entre ellas, mientras tanto la contabilidad le suministra información financiera confiable, que a su vez permita un aseguramiento y una confidencialidad de su información económica.

De acuerdo a Estrada (2015), sobre los sistemas de gestión contable:

Los sistemas de gestión contable bajo los elementos de la ética, la incertidumbre y la confianza, permiten asegurar la pertinencia del sistema de control de calidad en la actividad de la auditoría financiera mediante una relación de confianza pública y función social de las organizaciones y de los sujetos que las operan (p.1)

De acuerdo a muchos autores nos hacen referencia a que una buena gestión contable de una empresa hace reconocer todas las debilidades y fallas que puede poseer y así enfrentar esas eventualidades y dar propuestas a la gerencia para que así no afecte el rendimiento contable de la empresa, si mediante el buen uso de una buena gestión contable podemos planificar planes que sirvan para tomar una buena gestión del control interno mediante la documentación contable. La gestión contable se acopla mucho con buenos resultados que para el nivel gerencial es de gran agrado, para que manejen una información clara, concisa y eficaz que les permita tomar decisiones que puedan afectar la planificación, coordinación y evaluación empresarial.

Por tal razón la gestión contable es una herramienta vital en la empresa para que todos los departamentos y más para los gerentes, tomen dediciones para generar nuevas estrategias o planes de acción que lleven a la empresa a tener muy buenos resultados en el mercado, porque toda la gestión que se lleve en la empresa se va a ver reflejado en consolidar los nivel productivos financieramente por tal motivo una excelente gestión contable va blindar la empresa de todas herramientas que puedan fracasar o traer niveles negativos.

En este sentido, la contabilidad al ser tan importante en las empresas requiere que se haga de manera eficaz y esto se logra implementando un sistema de control y verificación de los procesos contables realizados por los distintos empleados, los errores de trascripción de información son comunes a la hora de visualizar los procesos y no sólo ocurren por la poca preparación en las áreas sino que también hay que tomar en cuenta que somos seres humanos y el margen de error se tiene que tomar en cuenta en cualquier aspecto así exista cualquier mecanismo que lo regule.

En otras palabras, los sistemas que manejan la información tienen que seguir ciertos lineamientos, que permitan a las auditorías externas e internas lo que debe realizar una 
empresa en mayor o menor medida para que se tenga confianza plena en la información, pues por medio de ellas se asegura el cumplimiento de los objetivos empresariales, generando informes que se entregan a los altos y medios dirigentes de la organización para la toma de decisiones. Entre los tipos de sistemas, se pueden mencionar los siguientes:

Sistema de control de lazo cerrado, Mantilla S. (2012) destaca que el control de lazo cerrado es aquel en cual la señal de salida tiene efecto directo sobre la acción de control. Se puede ver el control de manera que se puede reducir el error y llevar la salida de sistema al valor deseado. Se denomina también sistemas de control realimentado y constituye la medula teoría general del control.

Este proceso puede generar un mecanismo de sistema que puede llevar la entrada en el proceso para así luego determinar un control riguroso del proceso y verificar el sistema que se va a genera de manera inmediata todas las mediciones de los elementos para luego tener una salida efectiva y tener una retroalimentación cuya base es el mecanismo correctivo.

Sistema de control de lazo abierto, de acuerdo a Mantilla, S. (2012), el sistema de control de lazo abierto:

Este tipo de sistema que la salida no tiene ningún efecto sobre la acción de control, ni se mide la realimentación en comparación con la entrada, sólo puede usarse si la entrada y la salida son conocidas y si no hay una perturbación ni internas y externas. (p.82)

Este sistema que genera un proceso rápido porque no se sustenta de la teoría de control y puede generar una calibración de un requisito técnico para evaluar de manera rápida la información generada este sistema nos refleja una entrada y una retroalimentación que puede ser la señal de salida o una función de esta y su derivada.

Sistema de control adaptivo, Mantilla, S. (2012) destaca que un sistema de control adaptivo se considera:

Un sistema de lazo cerrado que de manera continua y automatizada mide las características dinámicas de la planta o el sistema objeto de control, la comparan con las características dinámicas deseadas y usa la diferencia para verificar los parámetros ajustables al sistema y así generar una señal de acondicionamiento. (p. 84).

Mediante este sistema permite llevar un control más riguroso de los procedimientos que se puedan llevar en una empresa para así verificar que todo esté en orden de manera correcta ya que identifica las entradas de la información financiera y posterior genera un determinado control para una fase llamada identificación o medición que evalúa todo el procedimiento y generar una salida exitosa.

Sistema de control de aprendizaje, de acuerdo a lo sostenido por Mantilla, S. (2012:84) el sistema de control de aprendizaje es considerado "...un sistema de lazo cerrado o como un sistema adaptivo mejorado teóricamente, pues para su análisis matemático y aplicación práctica aún existen muchos aspectos por resolver, debido a que la variable más importante es la capacidad del operador humano". Este sistema genera unas fases que se pueden aplicar en diferentes áreas ya que este maneja una función de tener una entrada de información para así después generar un proceso y posterior verificar la información ya procesada.

Es importante que los sistemas al estar al mando de los seres humanos tengan la posibilidad de aprender de sus errores, es decir, ante una variación de su desempeño respecto a los indicadores esperados, debe realizarse la 
medida correctiva para posteriormente ajustar su comportamiento para no volver a cometer dicha variación y cumplir con los objetivos empresariales. En este sentido, este sistema es el que más se ajusta para el desarrollo del sistema de control de compras y ventas.

Sin embargo, el poco conocimiento acerca de los sistemas de gestión ha generado no sólo múltiples pérdidas monetarias, sino también perdidas en la información que suele ser muchísimo más útil para los procesos, debido a que los datos utilizados no son los correctos o no están adaptados al presupuesto planteado por la gerencia. No existe una regulación acerca de los mismos, ya que surgen muchas fallas en cuanto a la filtración de información financiera y en términos contables los registros llevados han sido descuidados de años anteriores.

En este sentido, la tecnología puede brindar las herramientas necesarias para tener una gestión de información eficaz, pero de nada sirve si no se tiene un dominio en cuanto al manejo de esa información, siendo este el casos de la empresa Marcla, C.A, dedicada a la fabricación y distribución de calzados que tiene una amplia jerarquía y antigüedad en el mercado venezolano y así como también es conocida su marca de zapatos María Pizzola para el uso de las damas, la misma se encuentra ubicada en la Zona industrial norte, calle Michelena sector Flor Amarillo en la ciudad de Valencia estado Carabobo.

En la empresa se observan debilidades en el sistema de control de compras y ventas, ya que los registros y presupuestos no manejan la cantidad de materia prima en existencia influyendo esto en los procesos de compras y ventas, y a su vez en la toma de decisiones por parte de la gerencia, ya que no les permite tener conocimiento exacto de cuanta cantidad de producto (calzado) pueden sacar para cubrir la demanda que ejercen los distintos proveedores, quedando un grado de incertidumbre de saber si se llega o no a la meta planteada, simplemente se generan los productos hasta que se termine el material y queden residuos 0 productos defectuoso que a su vez son desperdiciados.

Este problema no sólo afecta directamente los procesos de compras y ventas, sino que le ocasiona a la empresa cuantiosas pérdidas de dinero en material y mano de obra que no es utilizada correctamente. Las organizaciones para afrontar estos cambios necesitan complementar sus procesos contables aplicando sistemas conociendo sus beneficios y utilidad.

El sistema contable de cualquier empresa debe ejecutar tres pasos básicos; los datos se deben registrar clasificar y resumir, sin embargo, el proceso contable involucra la comunicación a quienes estén interesados y la interpretación de la información contable para ayudar a la toma de decisiones comerciales.

De acuerdo con Mcleod (2000:43) un Sistema de Gestión Contable "es un conjunto de elementos encargado de ejecutar las aplicaciones de contabilidad de una organización, las cuales se caracterizan por un elevado volumen de procesamiento de datos que tienen como finalidad la toma de decisiones gerenciales".

De este modo, un sistema contable está formado por tres subsistemas principales: Los que surten pedidos de clientes, los que ordenan existencia de reabastecimientos y los que efectúan los procesos de contabilidad general. Sistemas que surten pedidos de clientes comprende los siguientes subsistemas:

1.- Captura de pedidos.

2.- Manejo de inventarios.

3.-Facturacion.

4.-Cuentas por cobrar. 
Sistemas que ordenan el reabastecimiento comprende los siguientes subsistemas:

1.-Compras.

2.-Recepcion

3.-Cuentas por pagar.

En este sentido, los sistemas que efectúan procesos de contabilidad generan los resultados de los sistemas anteriores, sirven de base para que la gerencia tome decisiones acerca del funcionamiento de la organización. Estos comprenden los estados financieros. Balance General, estados de resultados y estados de movimiento del efectivo.

Por lo tanto, el departamento de ventas y compras ejercen una rama de la empresa fundamental a la hora de sacar el producto al mercado ya que una parte se encarga de lo que significa la obtención de materia prima para la producción y otorgar los acondicionamientos necesarios para que todo fluya con normalidad y la parte de ventas abarque lo que son los procesos de recolección de proveedores y el manejo de requisiciones que son llevadas al departamento de producción para tomar en cuenta la cantidad de producto a generar.

De acuerdo a todo los antes señalado, se plantea la posibilidad poner en práctica un sistema de control de gestión para optimizar los procesos operativos del departamento de compras y ventas de la empresa Marcla, C.A.

\section{MATERIALES Y MÉTODO}

La presente investigación está basada en un enfoque cuantitativo, orientado a un proyecto factible; por lo tanto, se seguirá la secuencia diagnóstico, pronostico, para estudios en el campo de las ciencias administrativas, ya que consiste en elaborar una propuesta viable destinada a responder las necesidades específicas, determinadas a partir de una base de diagnóstico. En este sentido, la propuesta es vista como solución a los problemas que puedan existir en la realidad que se estudia. Se considera de diseño investigación descriptiva con diseño de campo, porque los datos se recogen directamente de la realidad.

La población fue de los 59 trabajadores de la empresa, en cuanto a la muestra se tomó de manera intencional y se constituyó sólo con los empleados de nivel gerencial vinculados al departamento de compras y ventas, departamento que ejerce funciones de operatividad.

En cuanto a las técnicas de recolección de la información, fue la encuesta con base en un cuestionario estructurado contentivo de veinte (20) ítems, que se aplicaron a muestra escogida para obtener información de carácter confidencial de cómo se manejan los controles y en tercer lugar una escala a los supervisores del departamento de venta y compras. La validez del instrumento se ejecutó a través del juicio de un experto. En el caso de la confiabilidad de los instrumentos se aplicó la Kuder Richardson para el cuestionario, obteniéndose un resultado de KR20 $=0,73$ (confiabilidad Alta)

\section{RESULTADOS Y DISCUSIÓN}

Los resultados de la aplicación del instrumento a la población estudiada arrojo que la empresa no posee un manual de procedimiento que regule $\mathrm{y}$ verifique los procesos de compras lo que refleja una ausencia de políticas respecto al control de gestión según lo que apreciamos en los encuestados. Adicionalmente se observó que algunos procesos tales como son: los análisis de compras y la verificación de la materia prima no se 
realizan de manera adecuada ni con los debidos procedimientos, no reflejan los formatos de acompañamiento de pedido.

Para Montoya A. (2002:45), "la gestión de compras ha pasado de ser considerada una actividad meramente administrativa a ser reconocida por algunas empresas como un elemento clave para mantener y mejorar la posición competitiva". Mediante los procesos de compra se ven muy interrelacionados muchos aspectos en una empresa tanto financiero, administrativo y personal porque cada uno de ellos influye mucho para que se pueda generar la actividad de la empresa.

Por su parte, Perdomo A. (1997:93) define control de compras como un: "Plan de organización entre el sistema de contabilidad, funciones de empleados y procesos coordinados, que tienen por objeto obtener información segura, oportuna y confiable, así como promover la eficacia de operaciones de adquisición o compra"

El mismo autor establece los siguientes principios:

1. Separación de funciones de adquisición, pago y registro de compras.

2. Ninguna persona que interviene en las compras debe tener acceso a los registros contables que controlen su actividad.

3. El trabajo de empleado que intervienen en las compras, será de complemento no de revisión.

4. La función del registro de operaciones de compras será exclusivo del departamento de contabilidad.

El control proporciona diversas ventajas. Las cuales reducen el riesgo de robo, ya que cada movimiento de elementos está respaldado por un comprobante interno firmado por el responsable. De igual forma, obtener con rapidez y exactitud la información de los materiales utilizados para evitar problemas ocasionados por sobrantes o faltantes de elementos, ya que pueden ser suministradas las cantidades solicitadas en los momentos oportunos.

De igual manera se puedo observar que el departamento de venta tiene debilidades en los procedimientos de ventas, y no se les brinda la debida capacitación a los vendedores para concretar el debido proceso, esto incide en la deficiencia en las ventas de la empresa la cual ocasiona retrasos en los pedidos que proporciona perdida de mercancías.

De acuerdo a Parra E. (2003), establece que las ventas son:

ciencias que se encarga del intercambio de un bien $y / o$ servicio equivalente previamente pactado de una unidad monetaria, con el fin de repercutir, por un lado, en el desarrollo y plusvalía de una organización y nación y, por otro, en la satisfacción de los requerimientos y necesidades del comprador. (p.33)

Según el autor, las ventas son la parte fundamental y esencial de cualquier empresa ya que es a través de éstas que las empresas crecen: Muchos dueños de empresas actúan como si las ventas no fuesen importantes para sus empresas, lo cual es completamente erróneo porque sin ventas, no puede existir la empresa

Es por ello, que los empresarios deben conocer que las ventas siempre serán los pilares de las empresas, que si no se tienen ventas, entonces no se crea un negocio. Y existe un sinfín de información sobre ventas para una empresa que se debe conocer así que es importante comenzar por conocer desde lo más básico hasta 
lo más complejo para desarrollar todo nuestro potencial en esta área.

En cuanto el control de las ventas, Parra E. (2002) destaca que las son:

Es la ciencia que se encarga del intercambio de un bien y/o servicio equivalente previamente pactado de una unidad monetaria, con el fin de repercutir, por un lado en el desarrollo y plusvalía de una organización y nación y por otro, en la satisfacción de los requerimientos y necesidades del comprador (p.33)

Es la última función del proceso de ventas, consiste en evaluar y controlar a la fuerza de ventas. Es en este punto que se evalúa a los vendedores y al proceso productivo para verificar se cumplen los objetivos de venta, políticas de administración de cuentas y los lineamientos para la creación del producto. Debe existir una buena planeación en el área de ventas para no ser víctimas de fraudes incluso de los mismos socios, y para que el control en el área sea más fácil y se cumplan los objetivos a alcanzar.

De esta manera, surge la necesidad de proponer Un sistema de control de gestión para optimizar los procesos operativos del departamento de compras y ventas de la empresa por medio de la configuración de un modelo viable, con la finalidad de organizar y promover un mejor entendimiento en los procedimientos en el área de compra y ventas.

\section{Propuesta}

El objetivo principal del sistema propuesto es transmitir a los empleados de la organización de los procesos operativos asociados al Departamento de compra y ventas de una forma detallada y precisa, que permitan dirigir las actividades que se realizan a diario en el departamento.
La presente sistematización de los procesos operativos en la gestión para optimizar los procesos operativos del departamento de compras y ventas de la empresa Marcla, C.A. es un instrumento que permite asegurar la disponibilidad y el uso racional de las compras y ventas, para garantizar la continuidad de la empresa en el tiempo. También propicia que el personal que labora en dicho departamento pueda desempeñar sus labores de forma eficiente, llevando de manera responsables los controles del departamento.

\section{Justificación}

Para toda organización es importante mantener un adecuado control de compras y ventas en una empresa, ya que esto permite tener el conocimiento de lo que se dispone para poder hacer frente a las diversas necesidades y obligaciones de la organización. En tal sentido, es importante que el trabajo se realice de manera sistemática y organizada, proporcionando en todo momento la información que se requiera.

\section{Fundamentación}

Las organizaciones deben llevar un adecuado control de compras, como de ventas bien sea de forma general o mediante métodos simples, así como también el rubro de mercancía; por lo que la sistematización de los procesos operativos permite realizar un control adecuado con miras a lograr los objetivos organizacionales. La presente propuesta se sustenta en el modelo de sistema viable, donde se identifican 2 sistemas denominados sistema de procedimientos de compras (figura 1) y sistema de procedimientos de ventas (figura 2) cada uno de estos se corresponde con las funciones de ejecución, coordinación, integración, planeación y política, respectivamente. 


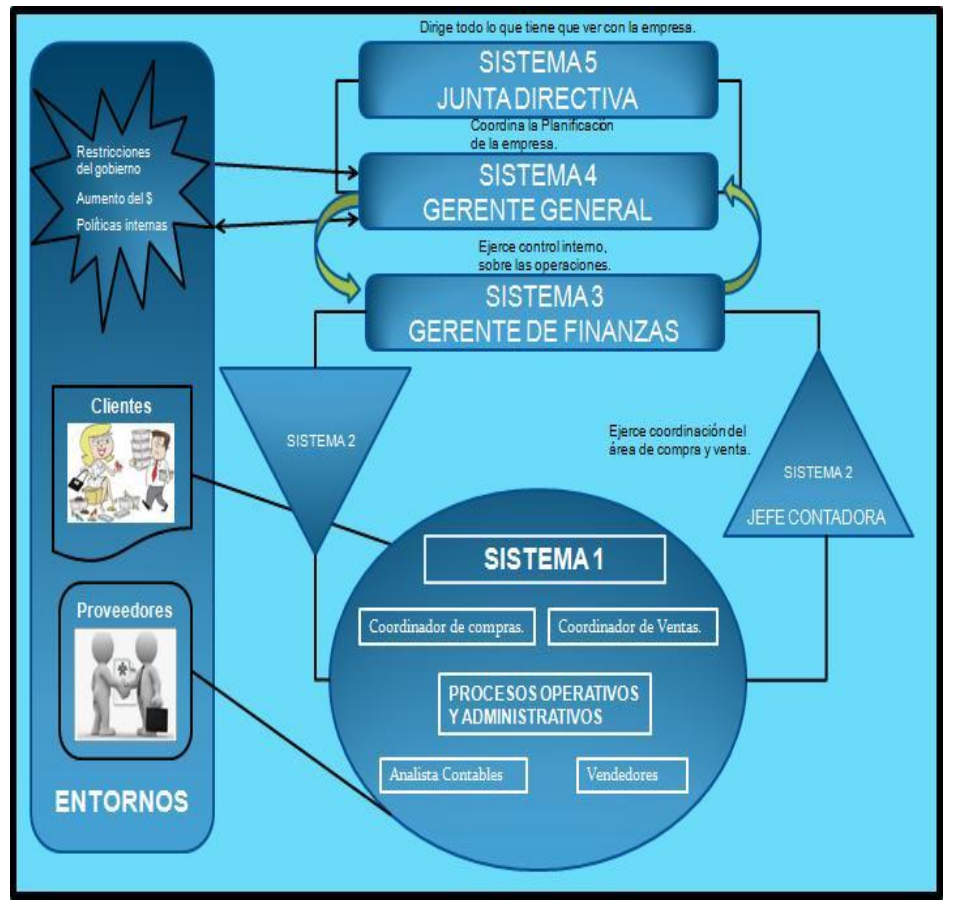

Figura1. Sistema viable del control de área de compras y ventas (Castellano, Madroñero 2017)

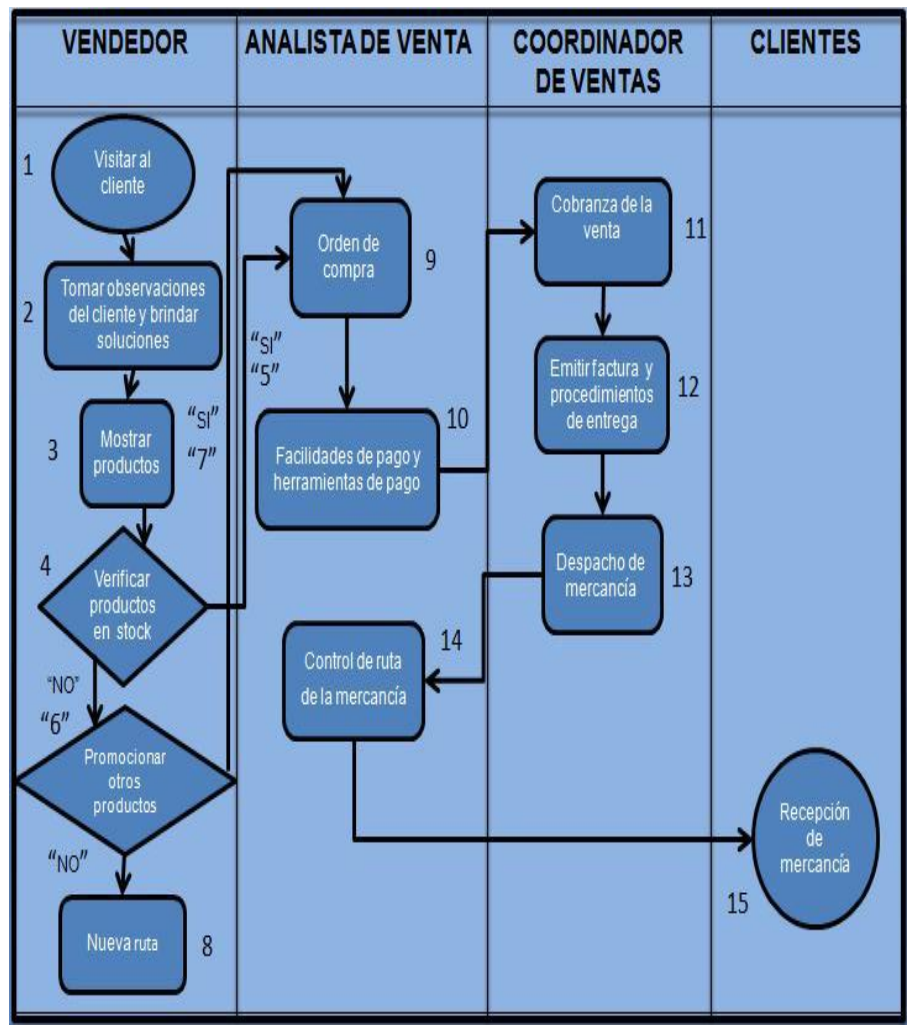

Figura2. Actividades del sistema de procedimientos de ventas (Castellano, Madroñero 2017) 


\section{Factibilidades}

Esto tiene que ver con la disposición de recursos, tanto financieros como estructurales. En virtud de la presente propuesta, a continuación, son planteadas las factibilidades de la misma:

\section{Factibilidad económica}

El presente trabajo se considera factible económicamente, pues la empresa puede asumir los costos derivados de la implementación de la misma.

\section{Factibilidad técnica}

La empresa cuenta con los equipos tecnológicos y la infraestructura necesaria para la adaptación de la propuesta.

\section{CONCLUSIONES}

De acuerdo a los resultados obtenidos con la aplicación del instrumento, a los fines de diagnosticar la operatividad de los procesos de compra y ventas de la empresa Marcla C.A. Se puede concluir que en la empresa se evidencia fuertes debilidades ya que no se cumplen con los procedimientos adecuados que permitan tener un adecuado control en las compras y ventas de sus producciones, teniendo como consecuencia que la empresa tenga perdidas en material y por ende de dinero.

Por todo lo señalado anteriormente, se plantea que la empresa Marcla. C.A. Implemente un modelo de sistema viable que le permita optimizar los procesos operativos en el departamento de compras y ventas. Así como establece controles en los departamentos de compra para tener una buena función de los diferentes procesos y llevar una debida ruta que con lleve a una buena gestión.

\section{REFERENCIAS}

Estrada, j. (2015). Sistemas de gestión de la calidad una herramienta imprescindible en la auditoría financiera [Articulo en línea] disponible en file://C:/Users/Adolfo/Downloads/297-5721SM\%20(1).pdf.[consulta:2017,Marzo:20]

Mantilla, S. (2012). Auditoria de información financiera. Bogotá 2009

Mc Leod, Raymond (2000). Sistema de Información Gerencial, 7ma Edición. México. Editorial: Pearson Educación con McLeod (2000).

Mcleod A (2000) control fundamentación de la gestión de los procesos y la calidad total editorial ESIC Madrid

Montoya, Alberto (2002) conceptos modernos de administración de compras editorial norma Bogotá

Parra E (2002) estrategias de ventas y negociaciones. Editorial panorama. México.

Parra, E. D. (2003). Estrategias de Ventas y Negociación. México: Panorama Editorial.

Perdomo A. (1997). Fundamentos del control interno. Ediciones contables, administrativas y fiscales S.A. México. Editorial Mc Graw Hill. [Consulta: 2017, Febrero: 15]

Perdomo, A. (2004), Fundamentos de Control Interno. (9ed). Editorial cengage learning Editores. 